\section{Cahiers de Narratologie}

Analyse et théorie narratives

$20 \mid 2011$

Voix off et narration cinématographique

\title{
Entre subjectivité et narration : la voix-off dans quelques documentaires français contemporains
}

\section{Antony Fiant}

\section{(2) OpenEdition}

\section{Journals}

Édition électronique

URL : http://journals.openedition.org/narratologie/6346

DOI : 10.4000/narratologie.6346

ISSN : 1765-307X

Éditeur

LIRCES

\section{Référence électronique}

Antony Fiant, «Entre subjectivité et narration : la voix-off dans quelques documentaires français contemporains », Cahiers de Narratologie [En ligne], 20 | 2011, mis en ligne le 18 juillet 2011, consulté le 21 décembre 2020. URL : http://journals.openedition.org/narratologie/6346 ; DOI : https://doi.org/ $10.4000 /$ narratologie.6346

Ce document a été généré automatiquement le 21 décembre 2020.

Article L.111-1 du Code de la propriété intellectuelle. 


\title{
Entre subjectivité et narration : la voix- off dans quelques documentaires français contemporains
}

\author{
Antony Fiant
}

1 L'histoire du cinéma documentaire est comme embarrassée de ses liens avec la voix-off, avec ce qui a longtemps été nommé « commentaire ». Celui-ci sclérose en quelque sorte et régulièrement toute audace esthétique ou dramaturgique, un peu à la manière dont a pu le faire le parlant dans le cinéma de fiction au cours des premières années 1930. Qu'on pense, par exemple, à la situation du documentaire entre l'essor du cinéma parlant justement, et celui du cinéma direct permettant la synchronisation entre image et son; une situation seulement sauvée par quelques nouveaux venus, notamment dans le documentaire français (Resnais, Marker, Rouch, Franju...), refusant les dimensions didactiques, propagandistes ou colonialistes impliquées par l'omniprésence du commentaire venant combler jusqu'à l'excès l'impossible synchronisme. Avec un tel héritage, rien d'étonnant donc à observer dans le documentaire contemporain un refus certain de la voix-off. De Frederick Wiseman à Sergueï Loznitsa, en passant par Wang Bing ou Gianfranco Rosi, nombre de documentaristes parmi les plus stimulants fuient ainsi délibérément toute forme de didactisme, la vocation soi-disant informative $\mathrm{du}$ documentaire, quand ça n'est pas une vocation propagandiste. Ils annihilent de cette manière la distance souvent crée par la voix-off et responsabilisent le spectateur invité à déduire les choses quand il n'est pas soumis à une expérience proprement poétique. En refusant voix-off ou commentaire, ces cinéastes contemporains tirent le documentaire vers le cinéma et l'éloignent du reportage, l'amalgame entre les deux n'ayant pas encore disparu, loin s'en faut, quand on observe le nombre croissant de films reposant sur des schémas bien rodés devenus archétypes d'un langage qui n'a plus grand-chose de cinématographique mais tout d'audiovisuel.

2 L'utilisation de la voix-off dans le cinéma documentaire n'est cependant pas condamnable, et sa persistance, au-delà d'une continuité didactique qui perdure, est aussi 
le signe d'un certain renouvellement. Pour tenter de le démontrer, disons que - et ce sera notre parti pris - la fonction narrative évidente, intrinsèque, de la voix-off doit être combinée avec une fonction subjective, se débarrassant ainsi du fameux anonymat de la voix-off en même temps que de son omniscience. On passerait alors du fameux nobody's point of view au somebody's point of view, de l'omniscience à l'expression de doutes. Nombreux demeurent donc les documentaristes contemporains utilisant la voix-off à des fins subjectives tout en demeurant narratives. Loin de toute perspective didactique et du commentaire "informatif», ils restituent ainsi une expérience toute personnelle du monde, ou d'un monde, en la proposant en partage au spectateur dans un rapport particulièrement intime. Entre voyages, enquêtes ou réminiscences, la voix-off ou voix-je (dans tous les cas étudiés ici, celle du cinéaste même) relève en effet de l'intimité (restitution d'un état d'esprit, d'une émotion, confession...) mais aussi de la narration en ce sens qu'elle organise le récit, influe souvent plus que les images sur celui-ci, retranscrit un itinéraire physique ou spirituel. À travers ces films et leurs voix, les cinéastes se racontent, s'inscrivent dans un questionnement existentiel, voire métaphysique.

Nous voudrions ici analyser la manière dont la voix-off participe de l'élaboration de nouvelles formes narratives, en l'occurrence documentaires, en trois temps, autour de deux fois deux films et d'une série de trois films, révélant trois types d'approches du réel : intimiste, historique et anthropologique.

\section{Voix-off et partages d'intimités : Là-bas de Chantal Akerman et Despuès de la Revoluciòn de Vincent Dieutre}

Nous croiserons ici, autant que faire se peut, deux tentatives de partage d'une intimité venant de la part de deux cinéastes qui, profitant de deux invitations à l'étranger pour donner des cours ou séminaires de cinéma, réalisent deux films qui, chacun à leur manière, font état d'une profonde introspection n'empêchant pas l'évocation de sujets plus larges. Là-bas de Chantal Akerman (production française), réalisé en 2006, témoigne d'une expérience de quelques semaines en Israël, à Tel Aviv, où la cinéaste séjourne dans un appartement qu'elle a loué. Elle n'en sort guère et le film est essentiellement constitué de plans tournés depuis le séjour de cet appartement, selon différents axes de prise de vue vers la fenêtre, obstrués par les lamelles d'un store, laissant deviner ce qui se passe aux fenêtres et aux terrasses des appartements d'en face et, plus rarement, avec quelques plongées, ce qui se passe dans la rue. Ce type de plan constitue à lui seul trois séquences d'une vingtaine de minutes entrecoupées de deux séquences d'environ cinq minutes, séquences de transition, respiratoires, faites de plans tournés sur la plage de Tel Aviv. Despuès de la Revoluciòn de Vincent Dieutre, réalisé en 2007, témoigne quant à lui d'une expérience de quelques semaines en Argentine, à Buenos Aires, où le cinéaste séjourne à l'hôtel. Contrairement à Akerman, Dieutre sort beaucoup et le film témoigne de ses déambulations diurnes, montrant abondamment les rues de la ville, ainsi que ses sorties nocturnes agrémentées de divers rapports sexuels avec des amants. Le montage du film, d'une durée de 55 minutes, beaucoup plus élaboré que celui du film d'Akerman qui ne compte qu'une cinquantaine de plans sur une durée de 80 minutes, repose sur une certaine accumulation, sur un flux - avec des phases d'accélérations et de ralentissements auxquelles la musique n'est pas étrangère -, ainsi que sur de nombreuses surimpressions ou lents fondus enchaînés. 
Qu'en est-il de la voix-off dans ces deux films ? Comment le récit dicte-t-il ou subit-il la présence de la voix-off? Comment mesurer son pouvoir sur le déroulement du récit? Quand intervient-elle dans le récit? Comment fonctionne-t-elle en termes de temporalité? Avant de tenter de répondre à ces questions, qui sont des questions formelles et relatives à une esthétique de la voix-off, il semble nécessaire de dévoiler les sujets traités par les voix-off d'Akerman et Dieutre. Dans une situation similaire, tous deux questionnent leur présent, convoquent le passé et au final se livrent à une profonde introspection. Akerman, après dix minutes sans paroles, lance son récit par la voix-off non pas sur les circonstances l'ayant amenée à Tel Aviv, mais avec l'évocation du suicide de la mère de l'écrivain israélien Amos $\mathrm{Oz}$ et de celui de sa tante Ruth, le premier, nous dit la voix, à Tel Aviv un jour de pluie, le second un jour de faible soleil à Bruxelles. À partir de là, Akerman, par l'intermédiaire de la voix-off, évoque tour à tour, dans des proportions variables et avec des liens évidents, l'histoire de sa tante Ruth, rescapée des camps de concentration nazis, sa propre enfance, la dépression dont elle est victime, Israël et sa situation (un attentat a eu lieu non loin de l'appartement), son travail de cinéaste à travers lectures et notes, la dimension matérielle de son séjour à Tel Aviv. Dieutre, lui, évoque, là aussi tour à tour, dans des proportions variables et avec des liens évidents, sa découverte d'une ville jusqu'ici inconnue, le bonheur qu'elle lui procure instantanément, les retrouvailles avec son ancien amant Hugo, son passé parisien avec une communauté d'exilés argentins, le séminaire de cinéma pour lequel il est venu, ses relations avec un autre amant, son enfance.

Sur les questions formelles, observons tout d'abord une distinction importante quant à la personne utilisée par les deux cinéastes. Akerman utilise la première quand Dieutre utilise la deuxième personne du singulier. Voix-je contre voix-tu, donc. Dans le second cas, personne n'est dupe du fait que c'est lui-même qui se cache derrière le « tu », a fortiori pour qui connaît un tant soit peu l'œuvre antérieure éminemment autobiographique de Dieutre et le timbre spécifique de sa voix. Il n'empêche que ce choix provoque, à force de répétition, une inexorable distance, en n'écartant pas la possibilité que le «tu » puisse désigner quelqu'un d'autre (à la façon d'un film épistolaire) ou bien en distinguant le narrateur du protagoniste. Cet effet est pleinement assumé par Dieutre et il ne faut surtout pas y voir le geste d'un cinéaste qui se défile, car il est bien en adéquation avec la dimension quasiment onirique $\mathrm{du}$ film, en tous cas sensiblement envoûtante, très présente dans le montage image. À première vue, les images d'Akerman ne racontent rien. La mise en scène relève du dispositif, elle est minimale ; et on peut rappeler là son goût pour les installations. Sous différents angles, on distingue à travers fenêtre et stores des silhouettes d'hommes et de femmes se livrant à des activités quotidiennes, banales, comme arroser des plantes, fumer une cigarette, observer les alentours ou tout simplement prendre l'air. Ainsi suggère-t-elle, de très loin, des potentialités narratives ou fictionnelles, aussi minimales soient-elles (et c'est là à Jeanne Dielman que l'on peut penser). C'est donc la voix-off qui va assumer la part la plus évidente du récit, dans une apparente et trompeuse autonomie, selon des modalités assez peu variables dans ses huit interventions. Chacune dure de deux à trois minutes - à l'exception d'une qui atteint six minutes - et elles se font d'une traite sauf deux, dans lesquelles la cinéaste marque une brève pause. Enfin, six de ces interventions se font sur un unique plan, deux autres sont découpées, respectivement en cinq et deux plans. 


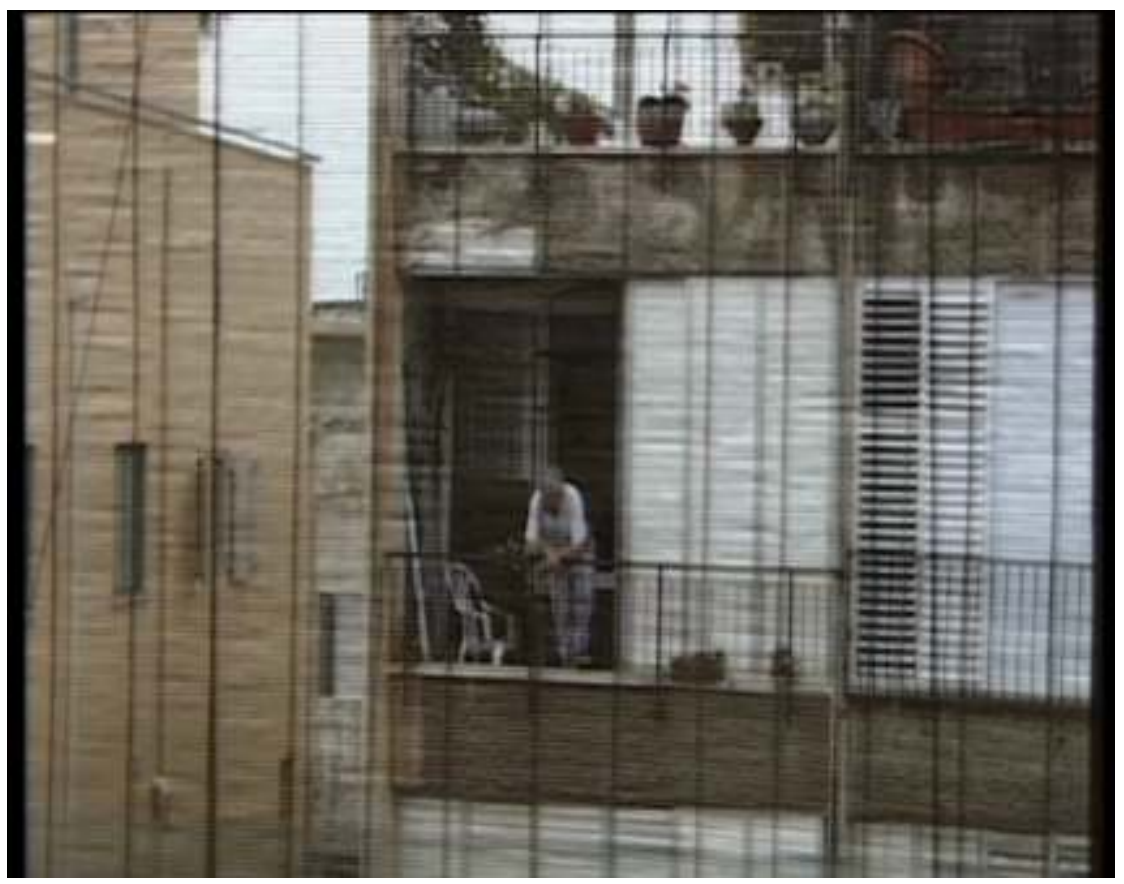

Une vue depuis l'appartement de Chantal Akerman dans Là-bas

[«Là-bas », production : AMIP / Paradise Films. Tous droits réservés.]

Chaque intervention de la voix, très tranchée, est précédée d'un long moment de silence et s'achève de même. Elles n'impliquent donc pas un montage image spécifique, y compris dans les deux cas constitués de plusieurs plans. Très vite, la voix-off prend le récit à son compte quand l'image est soumise à une opération de soustraction (axes de prises de vues limitées, montage limité, entrées dans le champ de la caméra limité à quelques amorces ou silhouettes dans des miroirs...). Cela ne provoque cependant pas de déconnexion. Au contraire, émane de ces partis-pris une vraie proximité spatiale et temporelle. C'est-àdire que cette voix-off est toute proche, qu'elle pourrait presque être considérée comme une voix hors-champ, qu'elle restitue une expérience toute récente, et l'on n'est pas si loin, du coup, d'une certaine simultanéité. D'ailleurs, la voix-off cède parfois le terrain sonore à une voix hors-champ quand la cinéaste reçoit des coups de téléphone. Le seul recul que l'on peut lui attribuer est relatif à l'écriture, à la qualité d'expression quasi littéraire empêchant toute dimension d'improvisation. Le texte de la voix-off jouant de l'explicite et de l'implicite, de la confession et de la pudeur, du matériel et du spirituel, du sérieux et du futile, de la souffrance et de l'humour, revient souvent sur des termes précis comme « là-bas » ou « place ». Dans les deux cas, c'est une question d'espace qui est posée et que le film dans son entier n'a de cesse de décliner. Car le Là-bas du titre ne désigne pas seulement Israël, c'est aussi, par exemple, cet ailleurs que scrutent à l'horizon nombre de passants devant la mer dans les deux séquences de transition. Comme le dit la cinéaste dans un entretien: «Israël n'est pas, ne peut pas être la solution[...] Cela veut dire qu'il n'y a peut-être pas de solution. Il n'y a pas de «là-bas ", pas de paradis, pas d'ailleurs.[...] Ce n'est à jamais qu'un rêve, ce «là-bas ${ }^{1}$ ». » Aussi en revient-on là très clairement à l'interrogation d'ordre existentiel récurrente dans le film : « Quelle place?».

8 Les choses sont en quelque sorte moins tranchées dans le cas du film de Dieutre. La voixoff y est beaucoup plus présente, la dimension littéraire du texte beaucoup plus poussée. Le récit devient songerie, en parfaite adéquation avec le traitement des images. Surtout, la voix-off, bien qu'omniprésente, refuse les pleins pouvoirs. Elle s'efface régulièrement 
au profit de quelques passages "dialogués", mais surtout ses interventions sont marquées par des ouvertures en crescendo et des fins en decrescendo. Comme si elle n'était pas si importante ou indispensable que cela, comme s'il n'en restait que des fragments, comme si ce qu'il en reste dans le film reposait sur des choix arbitraires... bref, autant de manières d'y voir une nouvelle contestation de l'omniscience de la voix-off. La sortie du film, discrète, a suscité très peu de commentaires critiques. On peut cependant remarquer que Gérard Lefort dans Libération parle à très juste titre d'un texte « lu en voixoff par Dieutre, qui n'est pas un commentaire qui dit ce que l'on voit, mais une sorte de canne blanche [...]. C'est ainsi que parfois le son de cette voix faiblit, s'estompe et même sombre, esquif englouti par plus puissant que lui, l'océan des images ${ }^{2}$. » De plus, la voixoff est ici ou là relayée par une autre, celle d'une femme lisant en espagnol, sur des temps très brefs, des poèmes de Silviana Ocampo, Matilde Alba Swann, Roberto Juarroz ou Pablo Neruda, pour leur sonorité, rien que pour elle, sans recours aux sous-titres.

9 Au final, de l'utilisation de la deuxième personne du singulier au recours à ces poèmes, la voix-off prend le risque d'une légère distanciation. Mais elle n'empêche cependant pas le partage de l'intimité d'un cinéaste qui est constamment dans la mise en forme (à l'image) et dans la mise en récit (avec la voix-off).

\section{Voix-off et enquêtes historiques : Le Brahmane du Komintern de Vladimir Léon et No pasarán, album souvenir d'Henri-François Imbert}

10 Il s'agit là de deux films revenant sur des faits passés et surtout de deux films parvenant à " présentifier » des faits historiques, capables de tisser de plusieurs manières, notamment en ayant abondamment recours à la voix-off, des correspondances ou liens étroits entre passé et présent. No pasarán, album souvenir d'Henri-François Imbert, réalisé en 2003, traite de l'exode vers la France des Républicains fuyant l'Espagne franquiste en 1939. Il le fait par le biais d'une collection de cartes postales représentant cet événement historique, collection incomplète que l'auteur consultait, enfant, chez ses grands-parents, et qu'il se propose de compléter en menant une enquête. Une bonne partie du film est réalisée au banc-titre, à partir des cartes postales en question. Le Brahmane du Komintern de Vladimir Léon, tourné entre 2002 et 2005, achevé en 2006 et sorti en salles en 2007, retrace, lui aussi sous la forme d'une enquête, l'itinéraire d'un révolutionnaire indien du nom de Manabendra Nath Roy (1887-1954). Elle aussi est déclenchée par un élément iconographique, en l'occurrence une photo prise le 17 juillet 1920 lors de l'ouverture du 2 ème congrès de l'Internationale communiste, montrant Roy au milieu de quelques-uns des plus célèbres dirigeants soviétiques d'alors: Lénine, Gorki, Zinoviev ou encore Boukharine.

11 En croisant là aussi les deux films, on peut observer notamment en quoi ils participent de la constitution d'une mémoire, et voir comment peut s'exercer, dans un geste finalement assez paradoxal, la subjectivité des cinéastes, principalement assumée par la voix-off, sur des "faits » historiques, dans un geste qui s'opposerait à l'objectivité recherchée par l'historien. Car ces deux démarches relèvent parfaitement de ce qu'Odette MartinezMaler et Laurent Véray résument ainsi dans une revue d'histoire à propos d'autres films que ceux-là : 
Il ne s'agit pas tant de chercher à s'affranchir d'une démarche dite classique ou traditionnelle d'écriture de l'histoire, que de trouver une forme adaptée à la spécificité du cinéma. En d'autres termes, il faut s'approprier les matériaux visuels et sonores, et par le montage, par le récit, leur donner une nouvelle cohérence afin d'en faire surgir l'historicité ${ }^{3}$.

Si les deux films sont des enquêtes impulsées par des documents iconographiques, si elles supposent des voyages, il n'en demeure pas moins qu'elles fonctionnent du point de vue de la dramaturgie de façons très différentes. L'enquête d'Imbert est comme recroquevillée sur les cartes postales omniprésentes à l'écran tandis que celle de Léon est entièrement bâtie sur ses différents voyages plus ou moins longuement restitués. Le recours à la voix-off est donc particulièrement favorisé par deux dispositifs qui pourtant s'opposent : fixité contre mouvement. Mais tous deux utilisent la première personne du singulier, celle des deux cinéastes qui n'ont aucunement recours à des émissaires, et la subjectivité est déjà et d'abord là, elle « s'incruste » dans les faits historiques.

L'un des aspects les plus passionnants des deux films, et qui en fait d'incontestables objets de cinéma, est qu'ils ne cachent pas les questionnements et les doutes qui animent les deux auteurs. Dès sa première intervention, Léon dit par exemple : «Je cherche moi aussi ma place, à la recherche d'un fantôme ». Alors que tout est réuni pour qu'ils restituent le résultat de leur enquête avec certitude et que l'écueil du didactisme leur tendait les bras (à travers une explication des cartes postales et un portrait sans failles du révolutionnaire indien), tous deux font le choix de l'expérience du monde, et prennent des risques, y compris celui de l'inaboutissement du projet. Cela n'était donc pas joué d'avance et les films gardent trace d'une part d'incertitude et de non-maitrise absolue. Voir ces deux cinéastes, et surtout les entendre en voix-off s'interroger sur la pertinence même de leur démarche, relancer de façon hasardeuse leur enquête - quand bien même on sait qu'ils n'ont retenu dans le montage final que ce qu'ils ont bien voulu retenir -, a quelque chose d'assez admirable qui atteste d'un geste créateur et non pas didactique. Imbert et Léon sont bien dans l'expression, dans une proposition qui est cinématographique avant d'être historique. Ils se livrent en pâture, loin de toute volonté purement informative. Là, la subjectivité prend le pas sur la narration, laquelle narration serait comme avérée, prise en charge par l'histoire qui se révèle peu à peu.

Dans No pasarán, les cartes sont d'emblée jugées mystérieuses puis, quand la collection se complète, Imbert observe qu'elles commencent à " raconter une histoire », rappelant au passage les puissances narratives, trop souvent négligées, du cinéma documentaire, a fortiori à partir de cartes postales. Pour autant, certaines cartes lui résistent, il avoue ne pas toutes les comprendre, certaines lui donnant une "impression d'irréalité » ne correspondant pas à sa connaissance des camps d'internement des réfugiés espagnols. En se lançant sur les traces d'un fantôme, Léon s'interroge clairement en voix-off tandis que l'image nous montre la photo originelle : «Qui était-il ? Comment était-il arrivé là ? Quel fut son rôle? Et pourquoi aujourd'hui plus personne ne semble s'en souvenir?» On tient là un questionnement classique chez quelqu'un se proposant de faire la lumière sur un personnage mal connu mais qui a manifestement tenu un rôle dans l'histoire de la diffusion de l'idéologie communiste. Le film ne répond pourtant pas à ce programme interrogatif, comblant certes quelques brèches dans l'itinéraire chaotique de son personnage, mais n'en livrant pas une image lisse, n'étouffant pas la parole de ceux qui contestent son importance. C'est notamment le cas d'une historienne indienne spécialiste des relations soviético-indiennes minimisant le rôle de Roy. Et puis, à la $95^{\text {ème }}$ minute $d u$ 
film, alors qu'il se trouve à Bénarès et qu'il y éprouve un sentiment "d'absence d'histoire », Léon fait le point sur son personnage :

Révolutionnaire trop mondain à Mexico, bolchevik trop incontrôlable à Moscou, communiste mais anti-stalinien à Berlin, membre du Parti du Congrès mais antinationaliste et anti-Gandhi en Inde, les positions de M.N. Roy l'ont toujours condamné à l'isolement. Cette solitude qui, si elle peut être celle des poètes, des philosophes, des fous, n'était sans doute pas compatible avec l'ample mouvement politique démocratique et radical que M.N. Roy envisageait de fonder à sa sortie de prison en 1936.

C'est toute la dimension métaphysique et existentielle de son itinéraire qui est proposée dans cette façon de préciser que Roy n'a jamais vraiment trouvé sa place. Reposant sur un personnage sincèrement désireux d'améliorer le monde dans lequel il vivait, le film est aussi une forme de constat d'échec. À propos de la voix-off de Vladimir Léon, remarquons qu'elle n'est pas là pour attester systématiquement une vérité. Comme le dit si bien Didier Péron :

Le commentaire, très écrit, articulé avec tout le recul que procure la lente élaboration d'un projet pensé, financé, monté pratiquement seul [...], creuse encore un peu plus la profondeur spleenétique, viscontienne du film, errance parmi les décombres du siècle ${ }^{4}$.

Dans le film d'Imbert, les témoins retrouvés par le cinéaste qui ont eu affaire avec la collection de cartes postales ne sont que rarement montrés mais plutôt évoqués par la voix-off. Le principe d'intériorisation du film - en même temps que centripète, tout nous ramenant aux cartes postales - trouve aussi à s'appliquer aux témoins, au détriment d'un rapport direct et révérencieux avec ceux-ci. C'est, par exemple, au début du film, l'évocation par la voix - tandis que l'image montre deux de ces plans de transitions sur un bord de mer puis se concentre sur des cartes de la série -, de la rencontre avec la veuve de l'auteur d'un livre intitulé Album souvenir de l'exil républicain espagnol en France, Juan Carasco, et qui lui confie des photos ayant servi à son élaboration, et ce, dans l'optique de continuer à transmettre la mémoire défendue par son mari. Un combat individuel pour une cause collective, une petite histoire au profit de la grande histoire, ainsi pourrait être résumée la façon d'intégrer ce témoignage ou plutôt cette passeuse de relais. Ainsi pourrait être aussi justifiée son absence à l'image. Car la cause est effectivement collective, appartient au registre public, historique. 


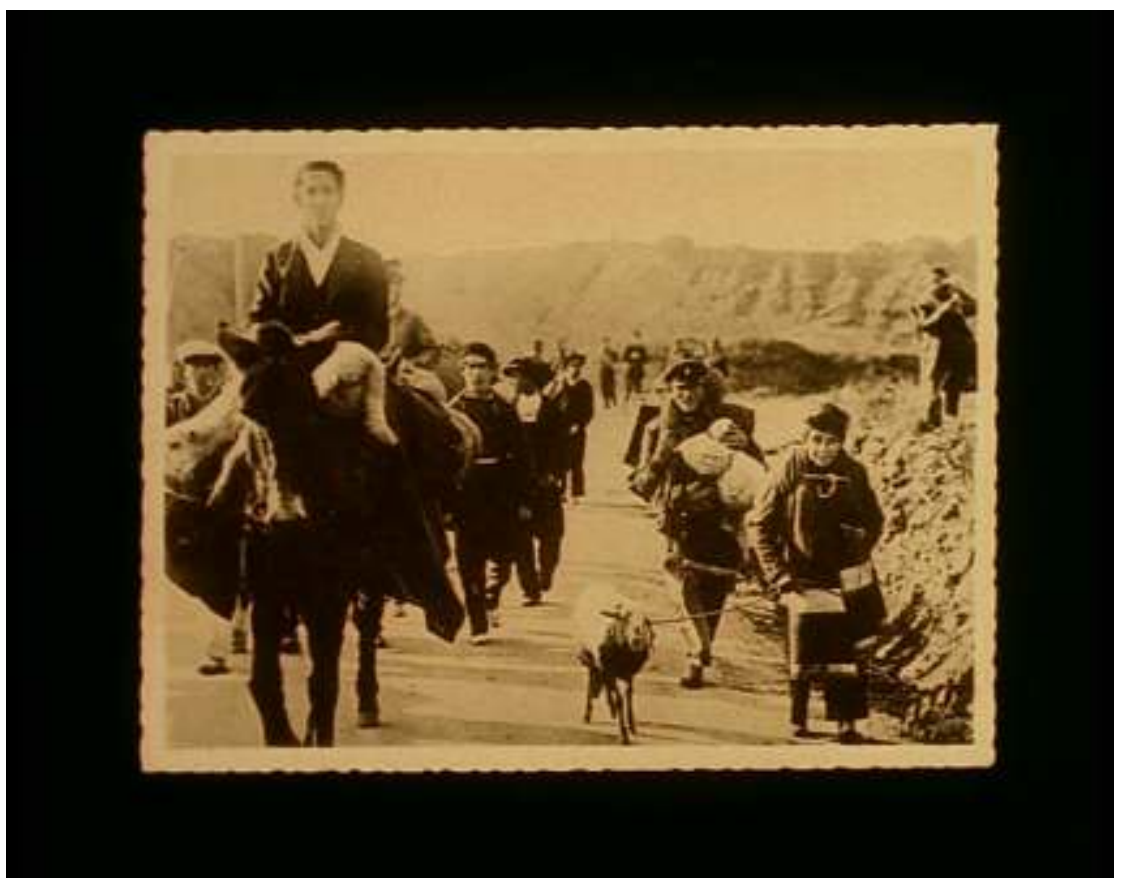

L'une des cartes postales retrouvées par Henri-François Imbert dans No pasarán, album souvenir [ "No Pasaràn, album souvenir », production : Centre National de la cinématographie / Libre cours Tous droits réservés.]

17 Pourtant, Imbert insère bien les témoignages de deux réfugiés espagnols de l'époque qui ont fait leur vie sur le territoire où ils ont échoué en 1939. Le premier se montre bien peu loquace. Le second témoignage, celui de Jo Villamosa, est certes plus fructueux mais on sent bien la volonté du cinéaste de ne pas laisser libre cours à la parole de son témoin. La première scène où il apparaît est découpée et ne dure pas plus de deux minutes. Il entame son récit sous les yeux de sa femme, présente dans le champ. Mais Imbert revient à deux reprises vers lui. La première fois pour évoquer des carnets de photo dont il lui avait parlé, avec quelques plans muets de l'homme dans son jardin couverts par la voix-off du cinéaste justifiant le retour chez son témoin. Cela dure une minute. La seconde fois, c'est juste pour évoquer, par voix-off interposée, une lettre reçue de Villamosa relative à une carte du camp de Gurs qui investit l'image. Le traitement de ce témoignage, son évolution (d'abord recueilli avec son et image, puis privé de parole, puis privé d'image) adoptent le mouvement du film, son écriture, basée sur l'image fixe et ce qu'elle a à dire, d'elle-même.

\section{Voix-off et regard anthropologique : série Profils paysans de Raymond Depardon}

18 Les trois films constituant la série Profils paysans - L'approche (2000), Le quotidien (2004) et La vie moderne (2008) -, sont le fruit d'un travail au long cours - plus d'une dizaine d'années - visant à montrer l'état du monde paysan en moyenne montagne, en Lozère, Haute Saône, Ardèche et Haute-Loire. Pour cela Depardon porte alternativement son attention sur des paysans en fin de vie (le terme de retraite n'a guère de sens) et de jeunes paysans. La volonté du cinéaste est de s'intéresser à une frange trop souvent oubliée de la population française, et selon les prévisions les plus pessimistes en voie de disparition. Témoigner, laisser une trace de ce monde dans une approche anthropologique est aussi vécu comme une dette de la part du cinéaste, lui-même issu de ce milieu quitté très tôt 
pour assouvir sa passion pour la photographie, avec la carrière que l'on sait. Il a souvent répété dans les entretiens accompagnant ces films que, puisqu'il n'avait pas su ou pu filmer son père paysan, il devait en quelque sorte se racheter.

Notons tout d'abord que le recours à la voix-off, ici important, est quelque chose de relativement récent dans son œuvre. La première période de celle-ci - disons pour faire court les années 1970 et 1980 - est très étroitement liée au cinéma direct, au mouvement, à la restitution d'itinéraires (que ce soit celui d'un homme politique en campagne, d'un reporter, d'une brigade de police ou d'urgentistes). Comme libéré de la fixité de la photographie dont il est issu, on a affaire à un cinéaste qui n'a de cesse de chercher sa place, et cela se traduit surtout, spatialement, par un cadrage en constant réajustement, par la recherche de la bonne distance. La seconde partie de l'œuvre - les années 1990 et 2000 - est plus posée, les mouvements de caméra plus rares, la caméra plus fixe, notamment dans ses films consacrés au monde de la justice et au monde paysan. Dès lors, la voix-off se fait plus présente et révèle un questionnement plus explicitement métaphysique, passant par la parole, exemplairement dans un film semblant avoir un tout autre sujet mais qui s'achève bien sur les racines paysannes du cinéaste, Afriques: comment ça va avec la douleur? (1996). Il ne s'agit dès lors plus de restituer un monde précis avec détachement, mais de se situer personnellement dans ce même monde.

C'est précisément ce qu'il fait dans les Profils paysans, en revenant constamment mais discrètement par la voix-off sur les relations qu'il tisse au long cours avec ces paysans (la subjectivité est là) et qui lui permettent de conclure dans son ultime intervention en parlant de l'un de ses personnages principaux : « Il sait que je n'ai plus peur de dire mon attachement à la terre des paysans. Apaisé, je retournerai aussi sur les hauts plateaux froids et les vallées profondes du massif. Ce soir, je filme cette lumière qui n'est pas comme les autres et je ne suis pas près de l'oublier.» Tout se passe donc comme si le cinéaste avait surmonté quelque chose avec cette expérience, qui a pour lui une incontestable valeur thérapeutique dans cette façon de renouer le lien rompu prématurément avec le monde paysan. Et si la première personne du singulier se manifeste dans ces quelques phrases, il convient de remarquer qu'il utilise essentiellement la première personne du pluriel tout au long du film, incluant ainsi sa collaboratrice et compagne Claudine Nougaret, responsable de l'enregistrement sonore.

21 On peut évoquer la voix-off dans cette série selon deux approches: ses fonctions et l'importance (au sens quantitatif) de sa présence. Outre d'évidentes fonctions introductives précisant le projet global dans sa toute première intervention et de tout aussi évidentes fonctions conclusives dans sa toute dernière intervention, on peut allouer quatre fonctions distinctes à la voix-off dans la série :

22 La présentation des personnages : lors de leur première apparition à l'image, Depardon présente ses nombreux personnages à l'attention du spectateur : nom, prénom, âge, lieu précis de résidence, lien de parenté avec un autre personnage, spécialité agricole sont ainsi soigneusement précisés. On imagine assez mal la plupart d'entre eux se présenter eux-mêmes, la pudeur n'étant pas la dernière de leurs caractéristiques. Un temps important s'écoulant entre chaque film, Depardon opère également de brefs rappels sur les personnages dans les $2^{\text {ème }}$ et $3^{\text {ème }}$ chapitres. Ainsi Depardon peut-il les inscrire rapidement dans un dispositif de recueil de la parole paysanne, son parti-pris n'étant pas de les montrer au travail mais de les amener à s'exprimer, dans ce lieu de prédilection qu'est la cuisine. 


\section{donc du théâtre, pour réaffirmer la capacité du cinéma à jouer des temps. Aussi, on tient} là l'indice ou le retour très bref de l'omniscience d'une voix-off émise depuis un temps autre et permettant recul ou anticipation. À la fin de L'approche,l'une de ces marques permet d'anticiper la mort de Louis Brès en ces termes et sur un plan le montrant dans la salle à manger d'un hôpital : "C'est la dernière fois que nous voyons Louis Brès, nous sommes le 9 février 2000, le 7 mars Louis Brès va mourir dans cet hôpital ». Puis, au début de Le quotidien, sur un plan montrant ce même Louis Brès en vie et à la porte de sa ferme, la voix-off revient sur le passé en ces termes : «Louis Brès, il avait été le héros de notre précédent chapitre, L'approche, nous avions été surpris par sa disparition et nous avions filmé son enterrement, ici, devant sa ferme, dans son pré, en présence de ces deux pasteurs venus d'Alès, de sa famille et de tous ses voisins. Marcel Privat du Villaret était là ». Où l'on voit que la voix-off retrouve là pleinement sa vocation narrative, à commencer par sa capacité de réagencement d'ordre temporel.

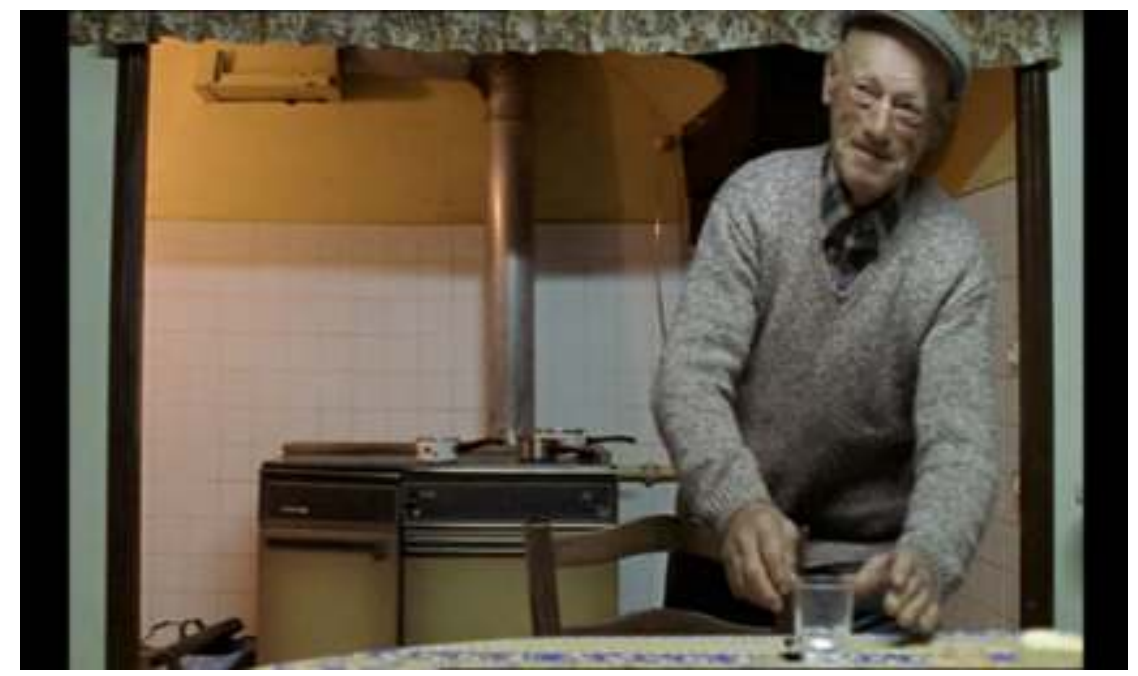

Raymond Privat dans Profils paysans, chapitre I : «L'approche » de Raymond Depardon [«Profils paysans : I'approche », production : Canal + / Palmeraie et désert. Tous droits réservés]

Sur l'importance (quantitative) de la présence de la voix-off sur les trois films (entre 80 et 90 minutes) le constat est simple : elle est de moins en moins présente.

Le titre du premier chapitre, L'approche, dit bien la patience dont a dû faire preuve le cinéaste pour faire parler les paysans mais aussi pour s'en faire accepter. Dans sa 
dimension anthropologique, la série révèle bien la conscience des paysans de vivre à part, selon des règles spécifiques, et la plupart rechignent à les exposer. Aussi, Depardon est-il accueilli avec une certaine méfiance qu'il devra vaincre. La parole et la confession mettront du temps à éclore et, dans un premier temps, c'est la voix-off qui vient combler les brèches pour caractériser les personnages. Selon les propres termes du cinéaste ${ }^{5}$ il use dans cette première partie d'une caméra « observante ».

Dès la seconde partie - Le quotidien - il passe en mode caméra "participante ». Les langues se délient (mais pas chez tous, l'un d'eux, Paul Argot demeure réticent); si la voix-off conserve les mêmes fonctions, elle cède plus rapidement la place à la parole, ses interventions sont moins nombreuses, leur durée est moindre. Les conversations avec un Depardon demeurant hors-champ se multiplient. C'est qu'il connaît maintenant bien ses personnages, le spectateur aussi. Il peut alors se contenter de rappels quant aux personnages, de précisions laconiques quant aux situations, de type : « Nous sommes en juin 2003, chez un notaire de Tence dans le département de la Haute-Loire. Nous retrouvons Amandine et Michel ».

Les interventions sont encore un peu moins nombreuses dans $L a$ vie moderne qui est un film de cinéma alors que les deux autres ont été conçus comme des films de télévision. Ce qui change, surtout, c'est que cette ultime partie sonne l'heure du bilan et qu'il n'est guère encourageant. Les plus âgés comme Marcel Privat, à bout de force, réduisent leur troupeau comme leurs activités tandis que les plus jeunes éprouvent les pires difficultés. La tonalité de la voix-off - non qu'elle soit joyeuse ou enthousiaste dans les deux premières parties - s'en ressent fortement.

31 Sans aller jusqu'à une proposition de typologie, la diversité des approches documentaires a primé ici afin de démontrer que c'est davantage dans ce registre-là que dans celui de la fiction que trouve à se renouveler et à se singulariser l'utilisation de la voix-off comme recours narratif. Finalement, plus que la subjectivité des cinq cinéastes dont il a été question, c'est leur sensibilité même qui, selon des modalités très différentes, est comme offerte en partage au spectateur. Ils y parviennent par le regard qu'ils portent sur leur sujet, un regard qui fait récit, mais un regard qui passe aussi par cet élément sonore qu'est la voix-off. Avec ces cinéastes, la voix-off conserve certes des capacités organisatrices au niveau du récit (des capacités narratives donc) mais divulgue aussi leurs « faiblesses », leur fragilité. C'est ce qui en fait le prix et la valeur.

\section{NOTES}

1. Laure Vermeersch, Pierre Zaoui et Zilberfarb, «Là-bas ou ailleurs. Entretien avec Chantal Akerman », Vacarme n 39, septembre 2007. En ligne : http://www.vacarme.org/article1288.html.

2. Gérard Lefort, «Quartiers de paume à Buenos Aires ", Libération du 28 avril 2010, p. VI.

3. Odette Martinez-Maler et Laurent Véray, «Éditorial », Matériaux pour l'histoire de notre temps n • 89-90, janvier-juin 2008, "Écritures filmiques du passé: archives, témoignages, montages », pp. 2-3.

4. Didier Péron, « Le rouge des Indes ", Libération du 24 octobre 2007, p. 26. 
5. "Séquences commentées par Raymond Depardon ", supplément de l'édition dvd de Profils paysans I et II, Arte Vidéo, 2006.

\section{RÉSUMÉS}

À travers l'analyse de sept films documentaires réalisés dans les années 2000 par Chantal Akerman, Vincent Dieutre, Vladimir Léon, Henri-François Imbert et Raymond Depardon, ce texte se propose d'étudier différentes modalités d'utilisation de la voix-off à des fins narratives mais aussi subjectives. Aussi, c'est la fameuse omniscience de la voix-off qui est remise en cause par ces cinéastes qui l'utilisent au contraire pour divulguer leurs doutes et leur fragilité. La voix-off participe alors de l'élaboration de nouvelles formes narratives, en l'occurrence documentaires.

INDEX

Mots-clés : anthropologie, documentaire, histoire, intimité, métaphysique, subjectivité

Index chronologique : 2000

Index géographique : Argentine, Espagne, France, Inde, Israël

\section{AUTEUR}

\section{ANTONY FIANT}

Antony Fiant est maître de conférences en études cinématographiques à l'université Rennes 2 et rattaché au laboratoire « La présence et l'image » (équipe d'accueil « Arts, pratiques et poétiques »). Il collabore à plusieurs revues de cinéma (Trafic, Positif et Images Documentaires) et est l'auteur de deux essais monographiques : (Et) Le cinéma d'Otar Iosseliani (fut) (2002, L'Âge d'Homme) et Le cinéma de Jia Zhang-ke. No future (made) in China (2009, Presses Universitaires de Rennes). 